\title{
Software and GNSS Fault-Monitoring for Automated Aircraft
}

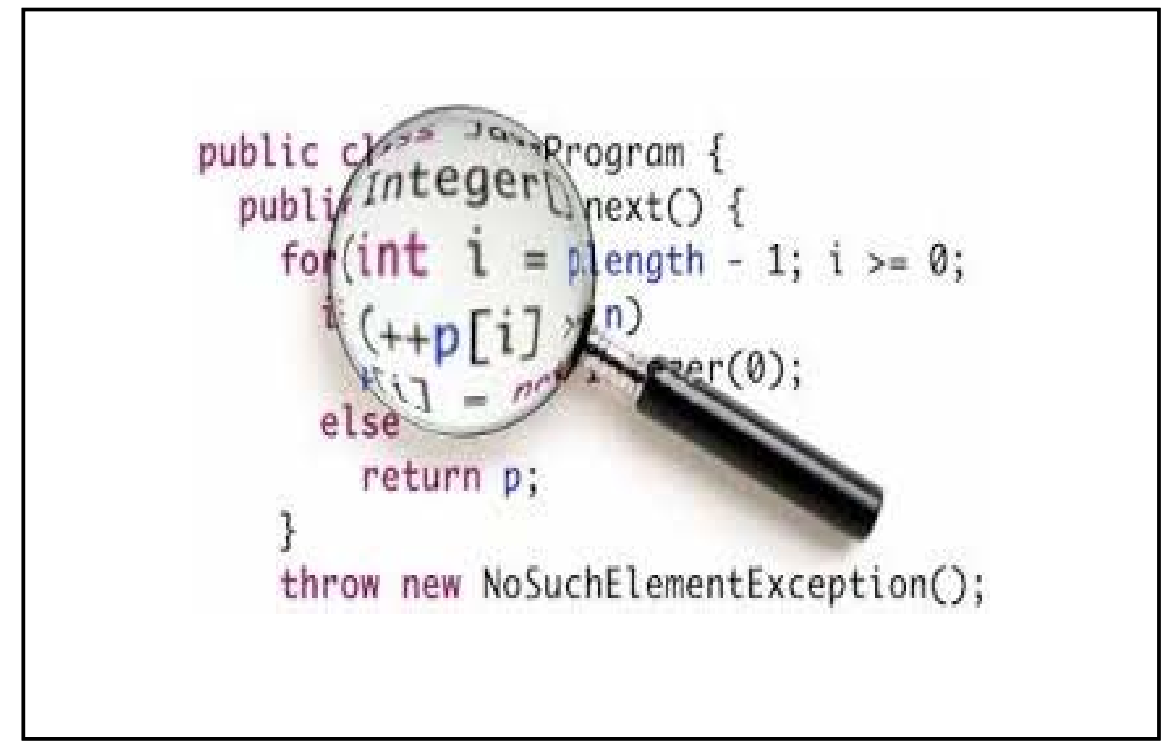

J.H. Rife, H. Huang, S.Z. Guyer

Tufts University

ION GNSS+ 2018

November 16, 2018

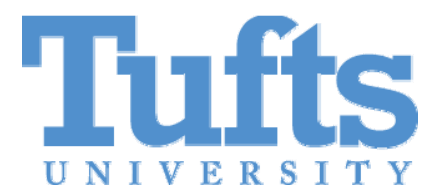




\section{Key Concepts}

- Propose an online bug-monitoring approach - Use analogy to GNSS augmentation system

- Discuss potential impact for pre-service verification 


\section{Aviation Software at a Crossroads}

Augustine's Law: Complexity of flight software grows an order of magnitude/decade

Will traditional verification approaches (e.g. RTCA DO-178) scale?

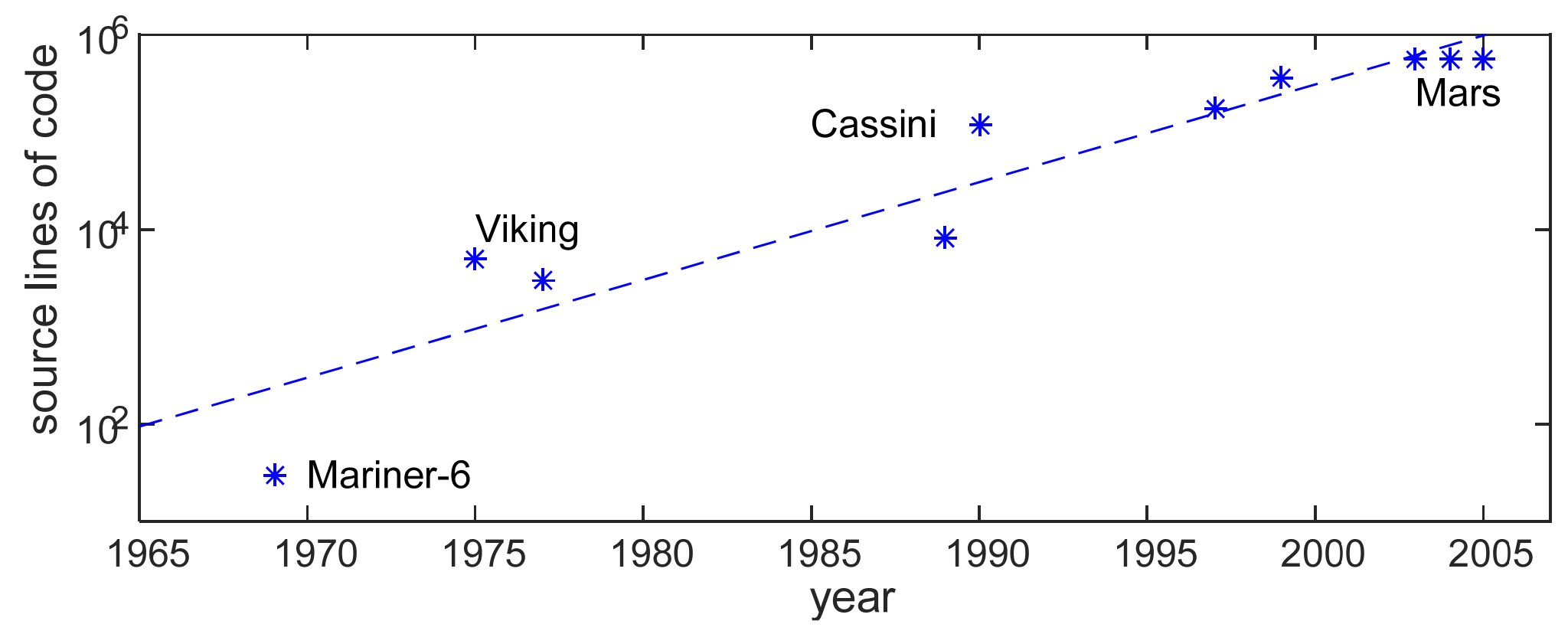

Figure adapted from Dvorak et al., NASA study on flight software complexity, 2009 
Aerospace, Meet Internet
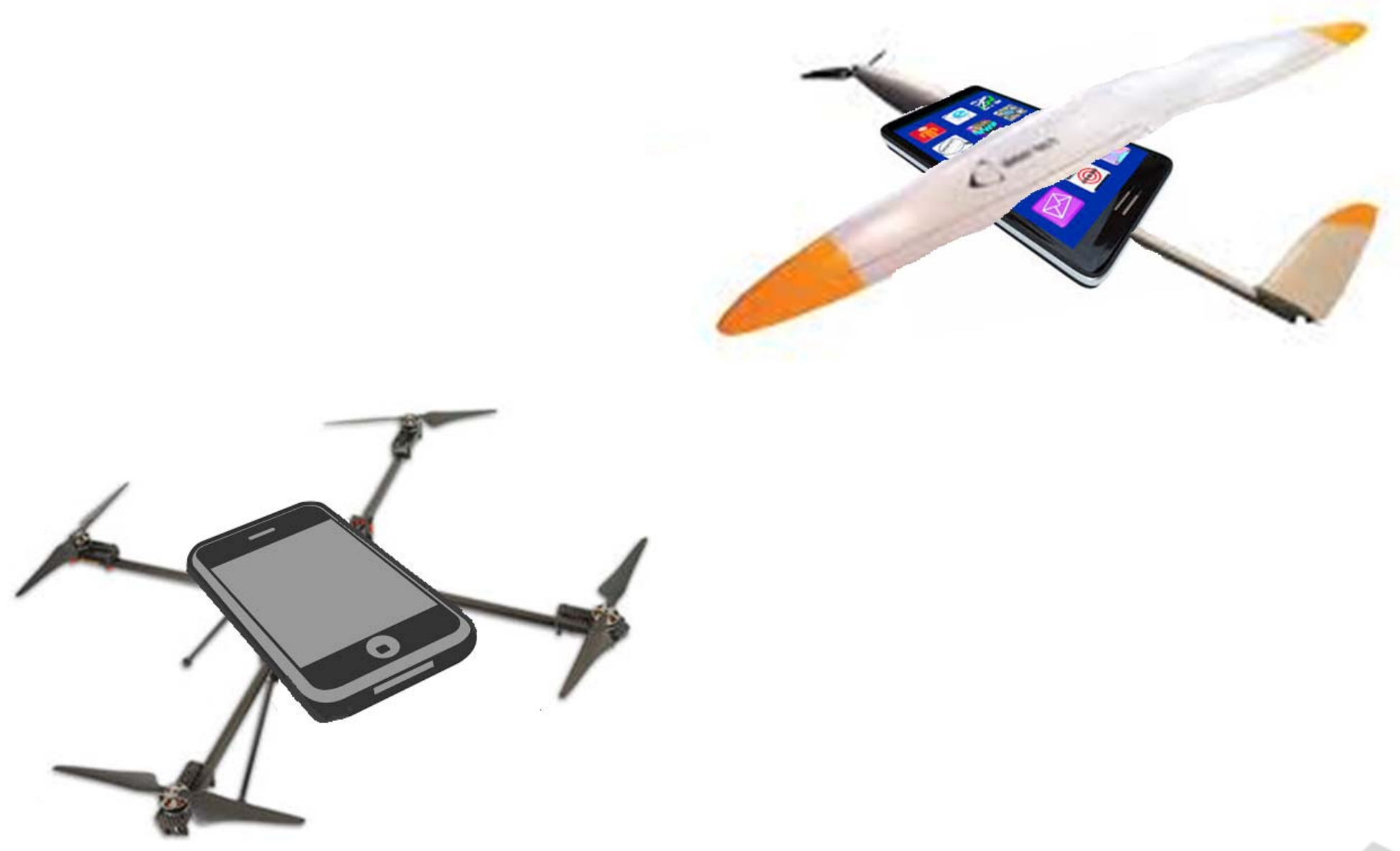

Tuff s 


\section{Continual Validation of Complex Systems}

Inspection,

Nondestructive Evaluation

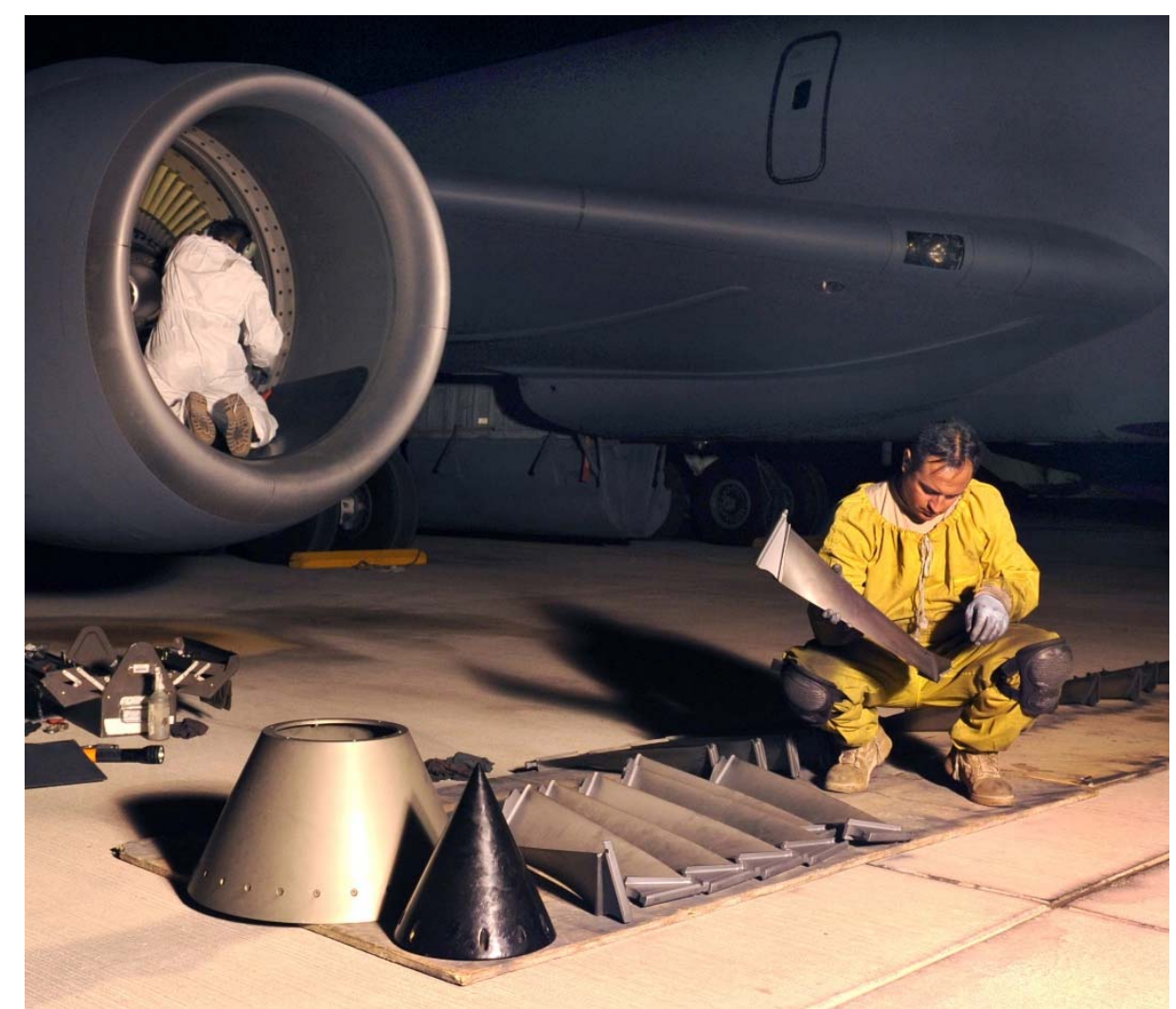

https://commons.wikimedia.org/wiki/File:Visual_inspection_of_compressor blades-090108-F-9919G-746.jpg
Monitoring of Navigation Signals (e.g. SBAS)

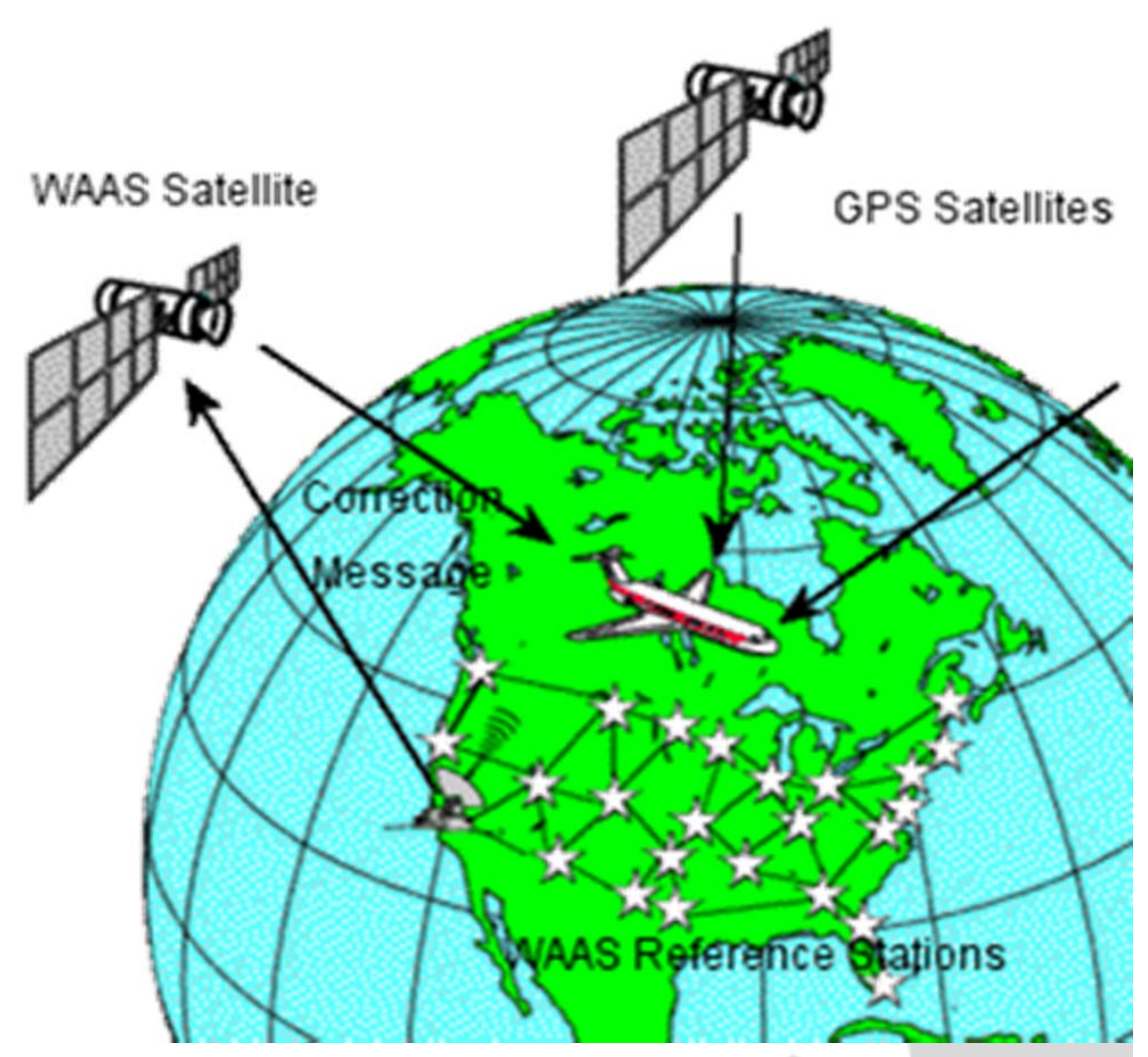

https://commons.wikimedia.org/wiki/ File:Waas.png 
Augmenting the Design Toolbox

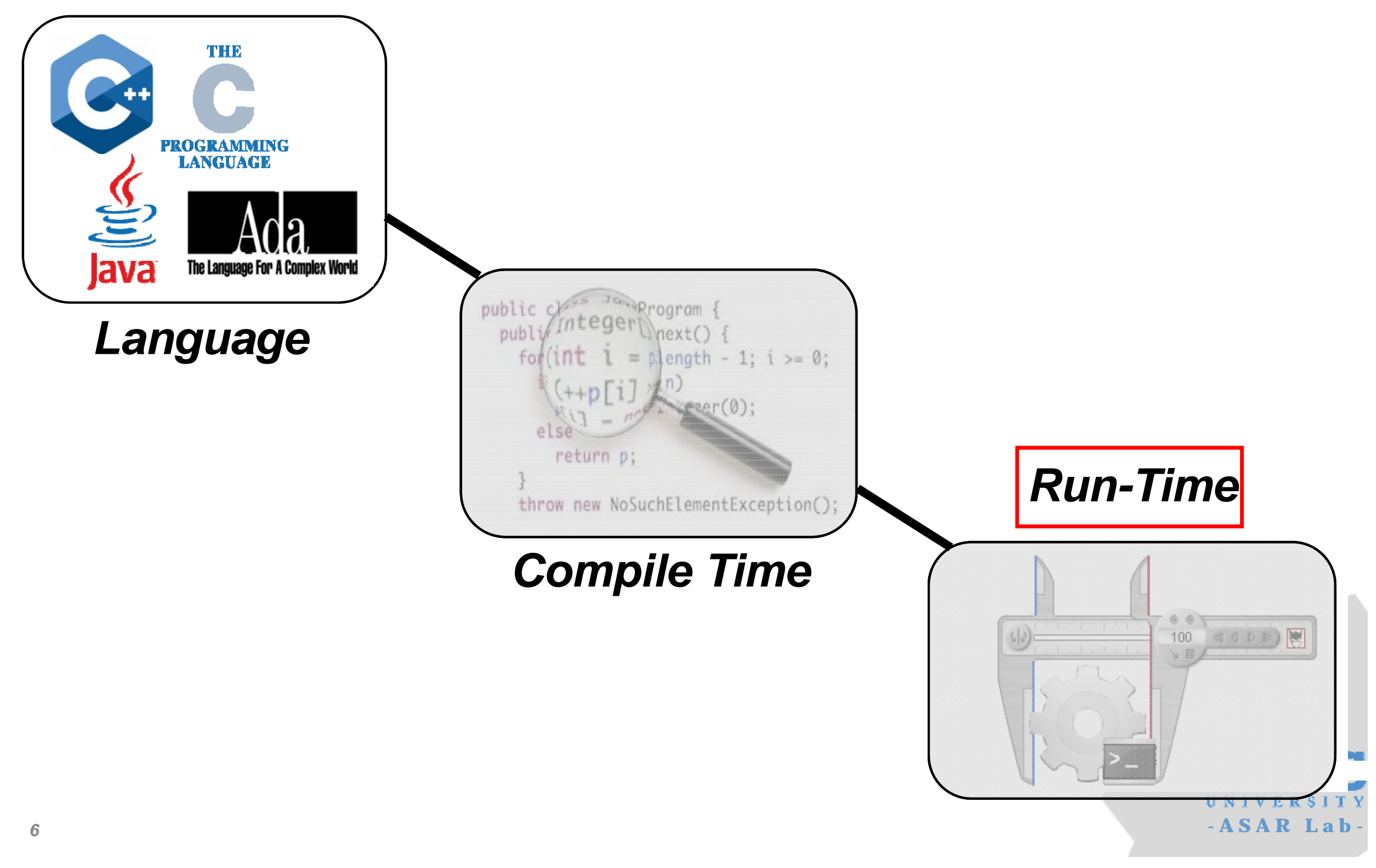


An Opportunity Exists

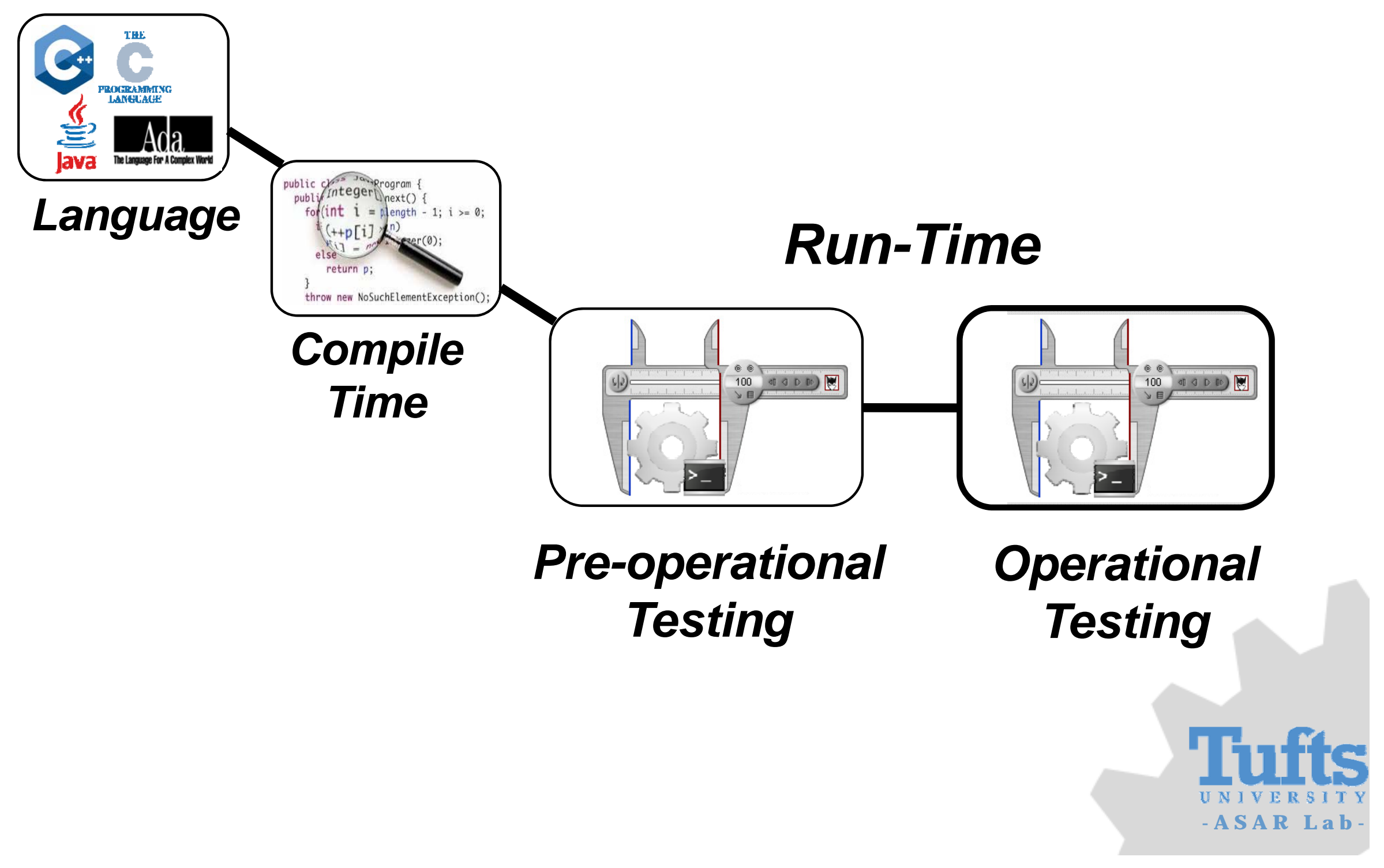




\section{Open Questions}

- How can we design a bug monitor analogous to monitors used for mechanical and electrical systems?

- How do we train a monitor to recognize what is normal behavior given that our software contains unknown bugs?

- How much can bug monitoring reduce the burden of pre-service verification?

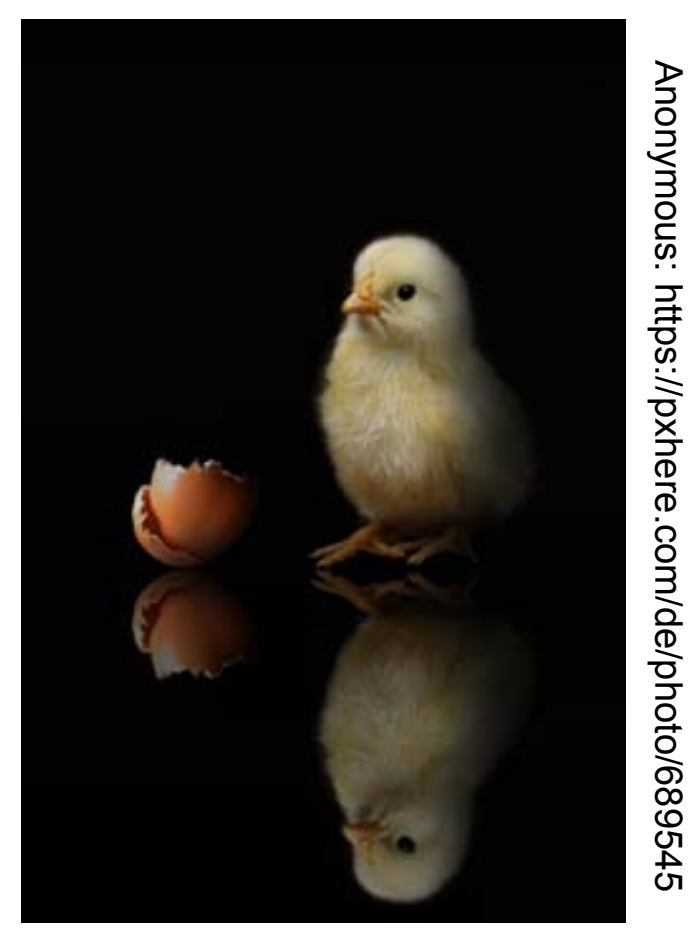




\section{Open Questions}

- How can we design a bug monitor analogous to monitors used for mechanical and electrical systems?

- How do we train a monitor to recognize what is normal behavior given that our software contains unknown bugs?

- How much can bug monitoring reduce the burden of pre-service verification?

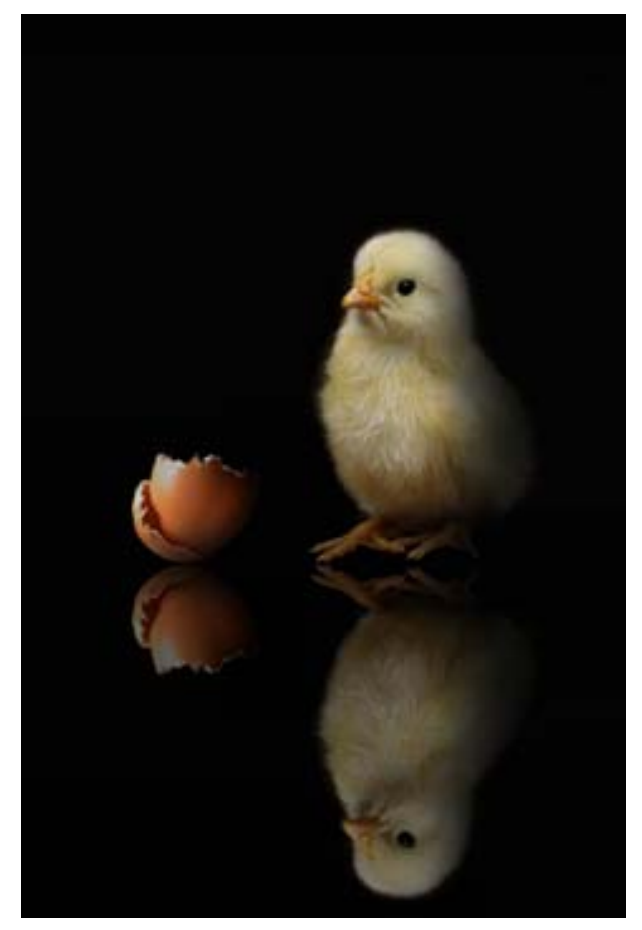


Monitor Design 


\section{Signal Deformation Monitoring (SDM)}

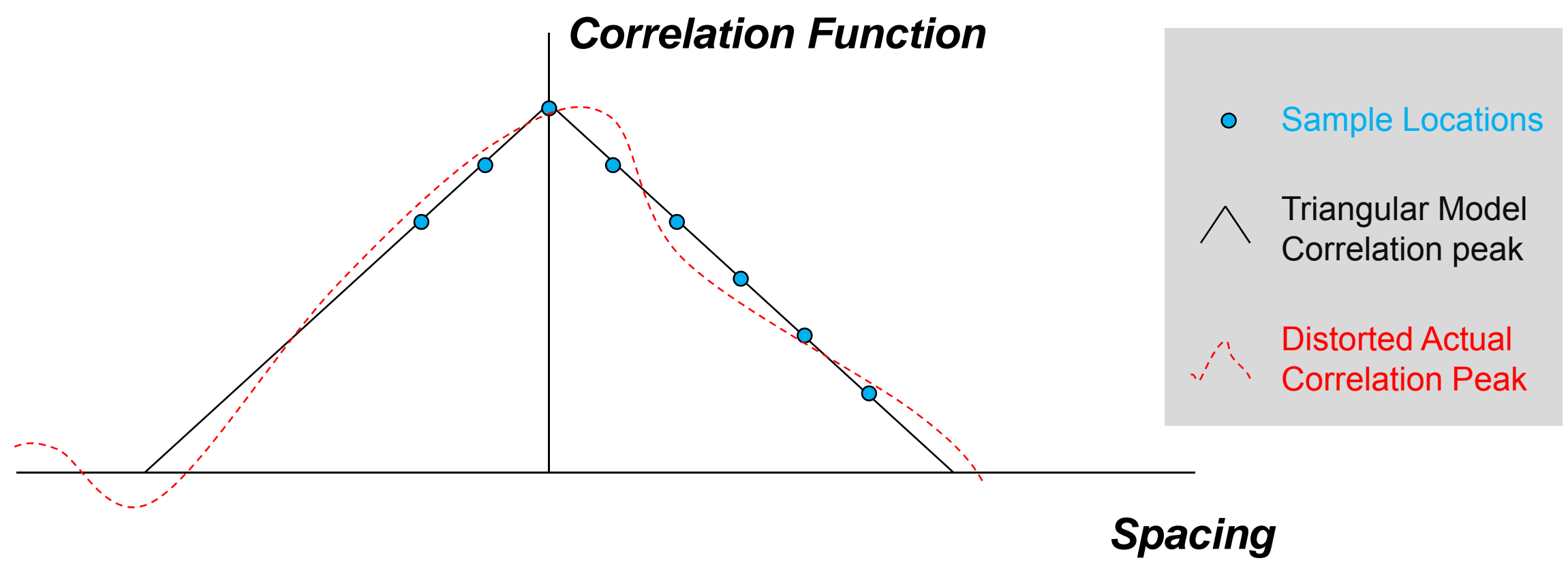

Sample correlation peak at 8 locations, Difference 7 neighboring pairs, and Compare to nominal values 


\section{SDM in GBAS}

The 7 errors (measured pairs less reference) are normalized and compiled in the vector $y$

$$
\begin{aligned}
& \mathbf{y} \in \mathbb{R}^{7} \longleftarrow \mathbf{y}^{T} \mathbf{y} \longleftarrow \begin{array}{l}
\text { Input signal vector has } \\
\text { expected value of zero and } \\
\text { unity variance }
\end{array} \\
& m \begin{array}{l}
\text { Test statistic }(m) \text { fuses input } \\
\text { signal values }(\mathbf{y})
\end{array} \\
& \text { alert if } m>T \longleftarrow \quad \begin{array}{l}
\text { Alert issued if test statistic }(m) \\
\text { exceeds threshold }(T)
\end{array}
\end{aligned}
$$


SDM (and other monitors) ensure integrity for safety-critical operations

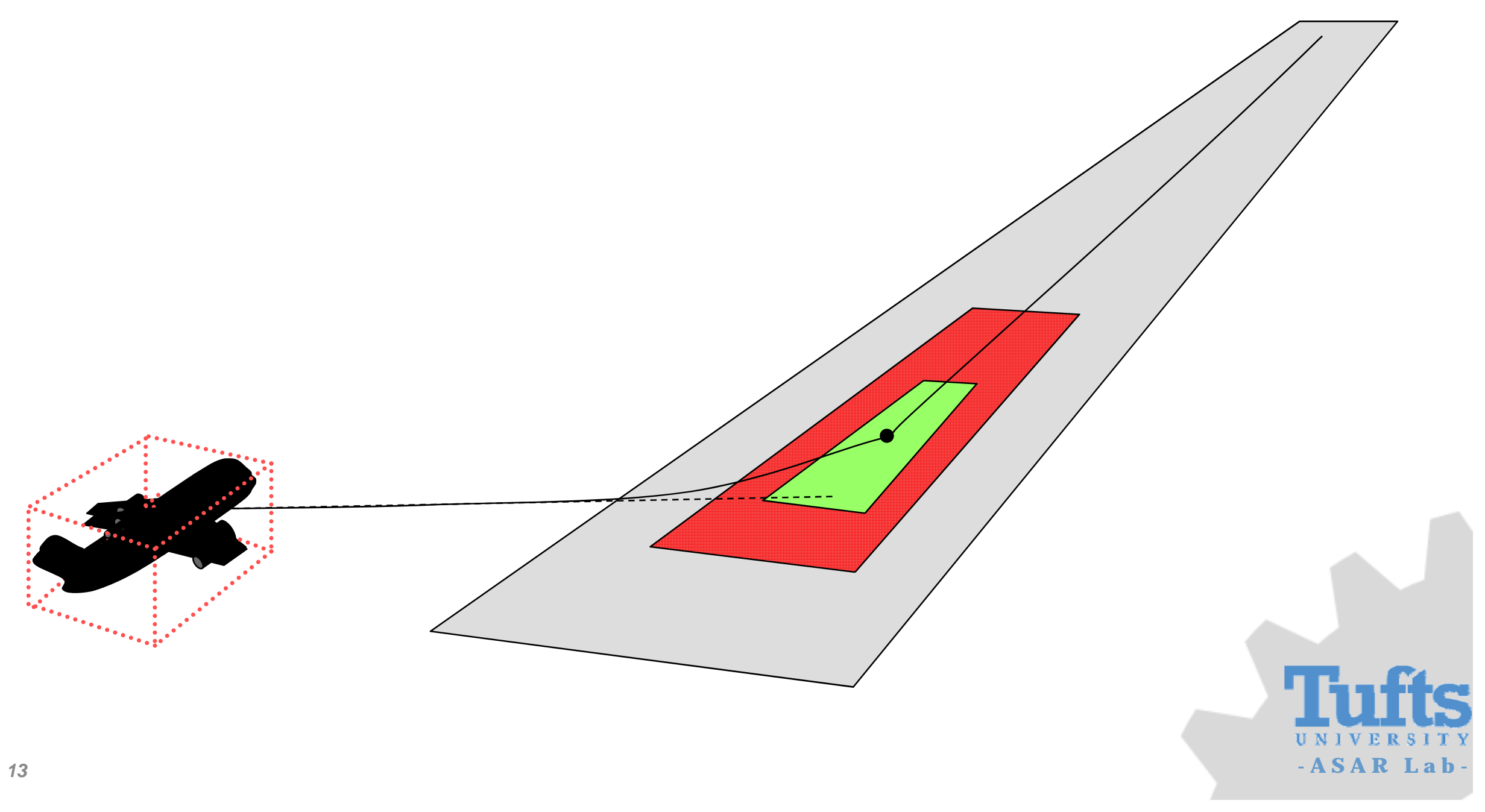


A Bug Monitor... 


\section{A Bug Monitor}

- Bug monitor looks for anomalous variable values

- Designer chooses a set of variables (or PROBES) to scan

- Choice of probes is critical

- Maximize sensitivity

- Minimize overhead

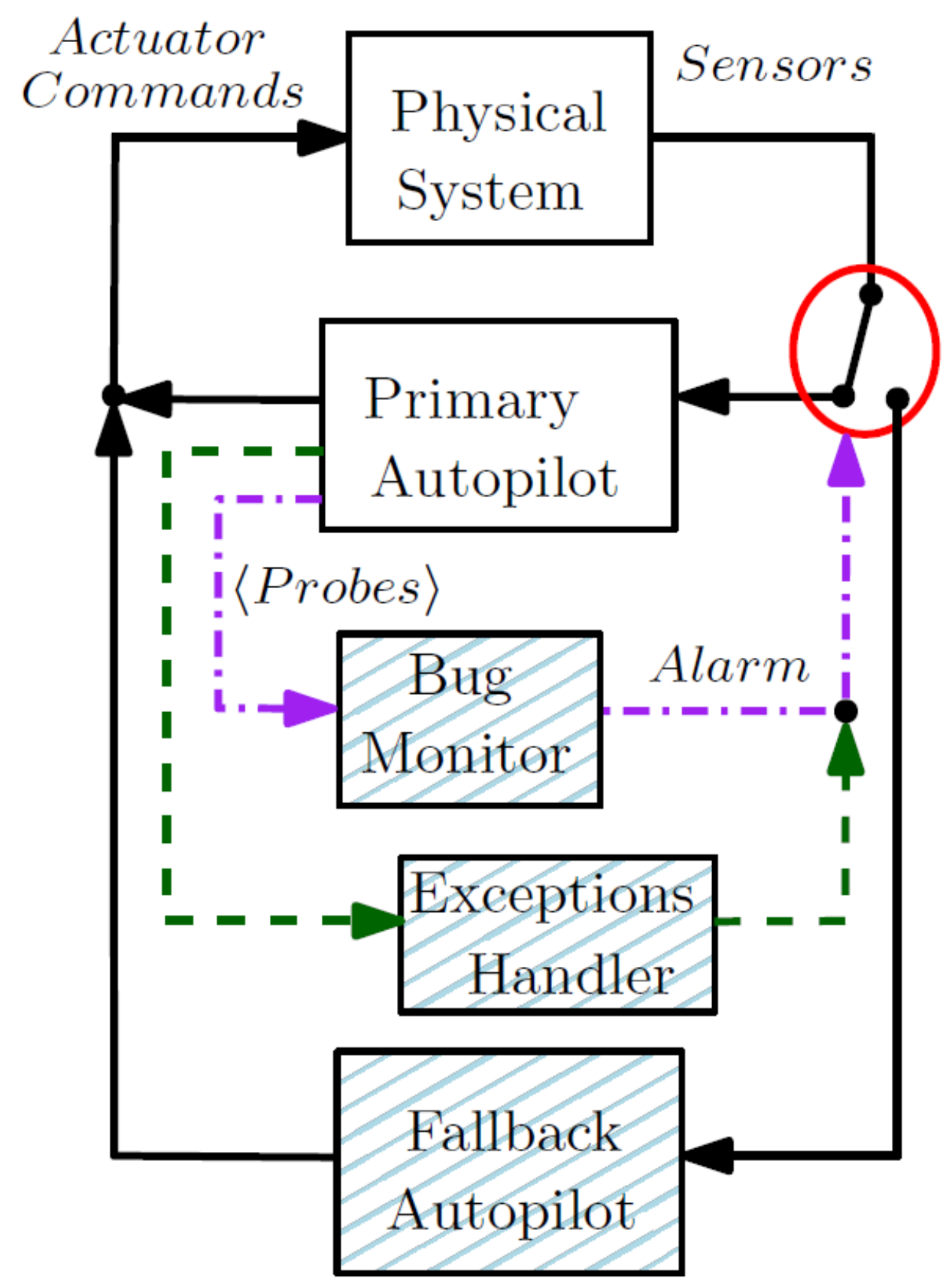




\title{
Implementation
}

\author{
Monitor Ardupilot +
} JSBSim flight simulator
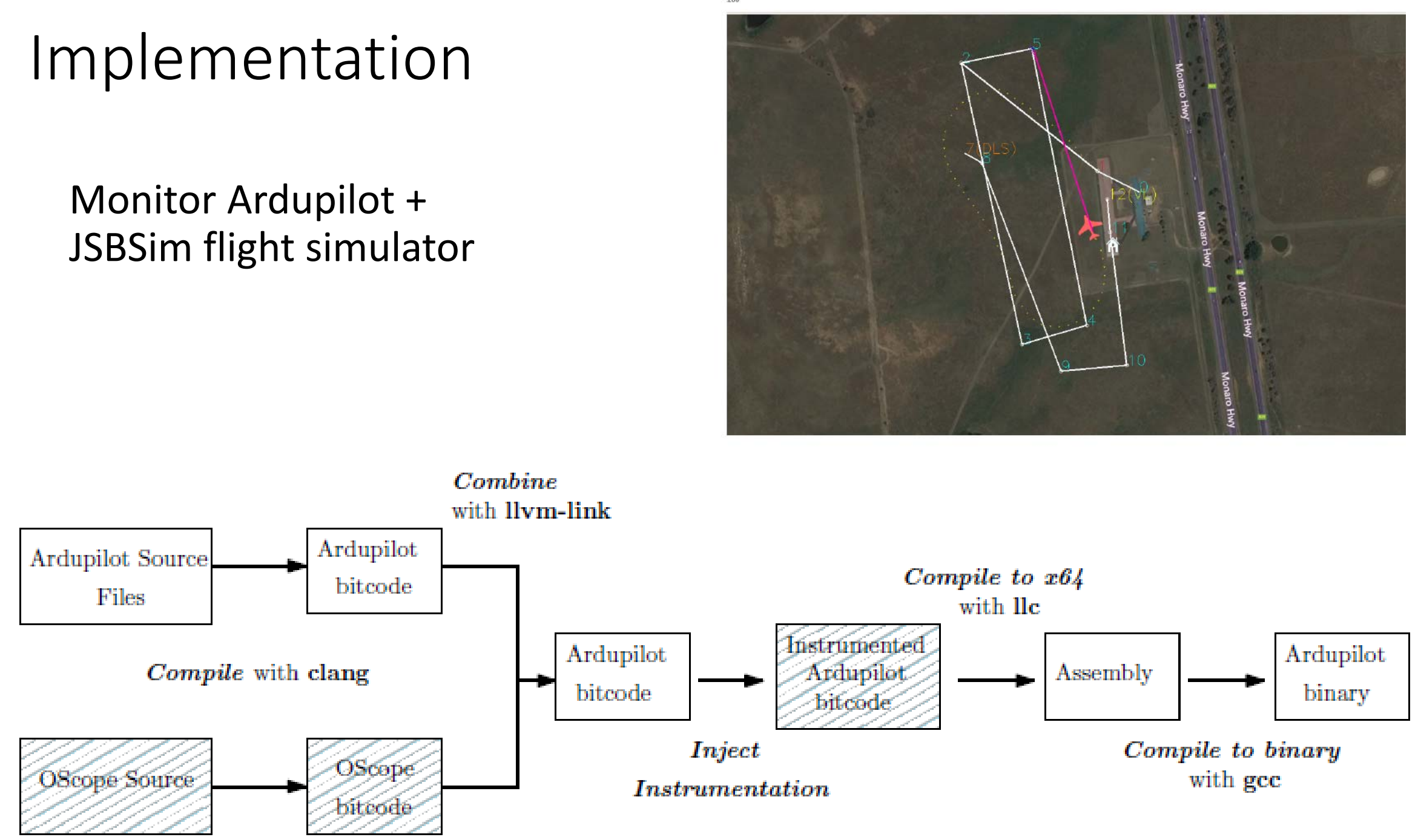


\section{Choice of Probes}

- In current work, consider two probe choices

- Random: Sets of 10 variables randomly selected

- Heuristic: Sensor/actuator interfaces with physical system

- In future work, seek to optimize probe selection

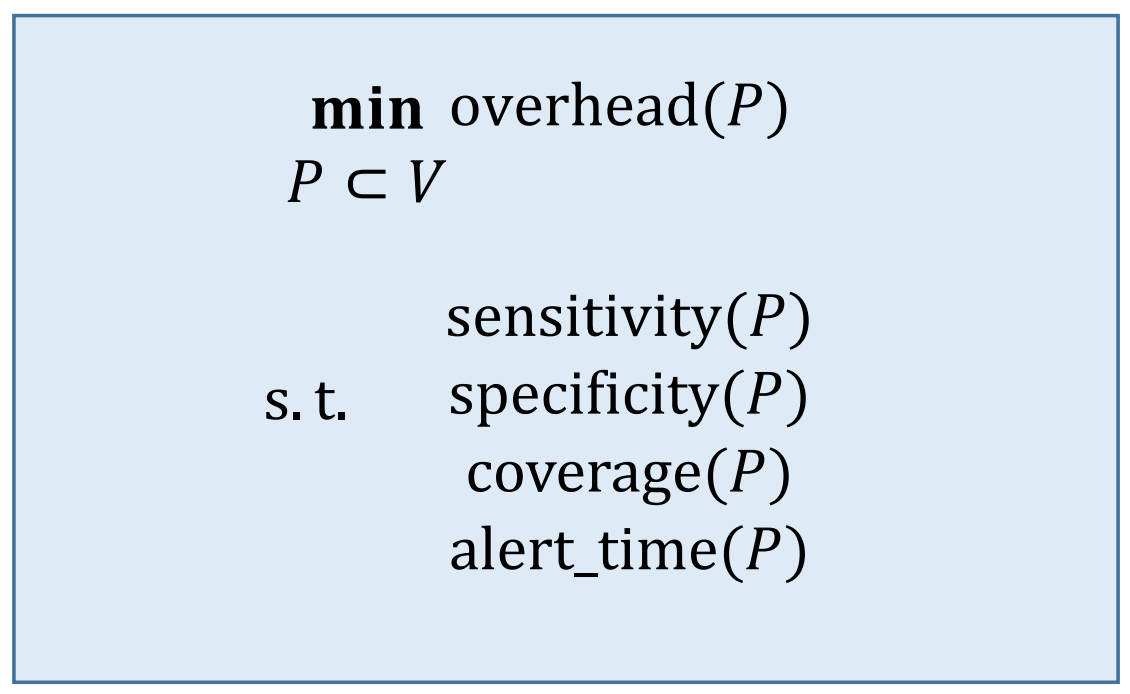


Safety Assurance 


\section{Open Questions}

- How can we design a bug monitor analogous to monitors used for mechanical and electrical systems?

- How do we train a monitor to recognize what is normal behavior given that our software contains unknown bugs?

- How much can bug monitoring reduce the burden of pre-service verification?

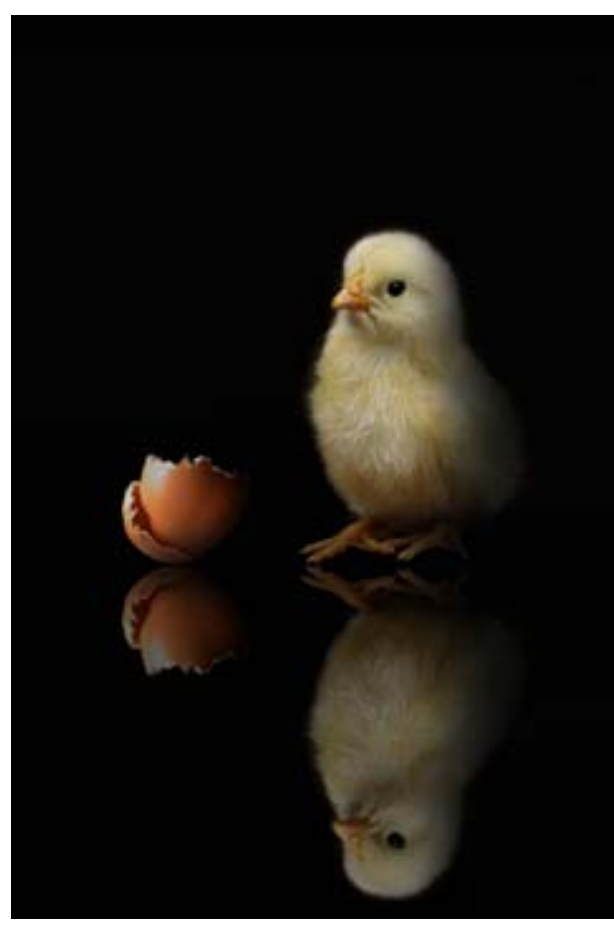




\section{Fault Tree Concepts: Single point of failure}

System failure due to hidden bug

\section{Pre-Service Verification \\ misses hazard}




\title{
Fault Tree Concepts: Single point of failure
}

\author{
System failure due to
}

hidden bug

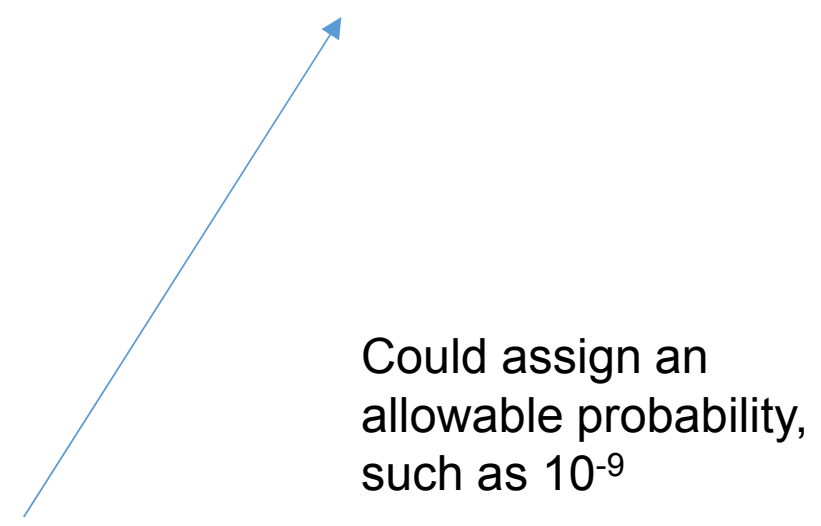

\section{Pre-Service}

Verification

misses

hazard 


\section{Fault Tree Concepts: Redundancy}

System failure due to

hidden bug

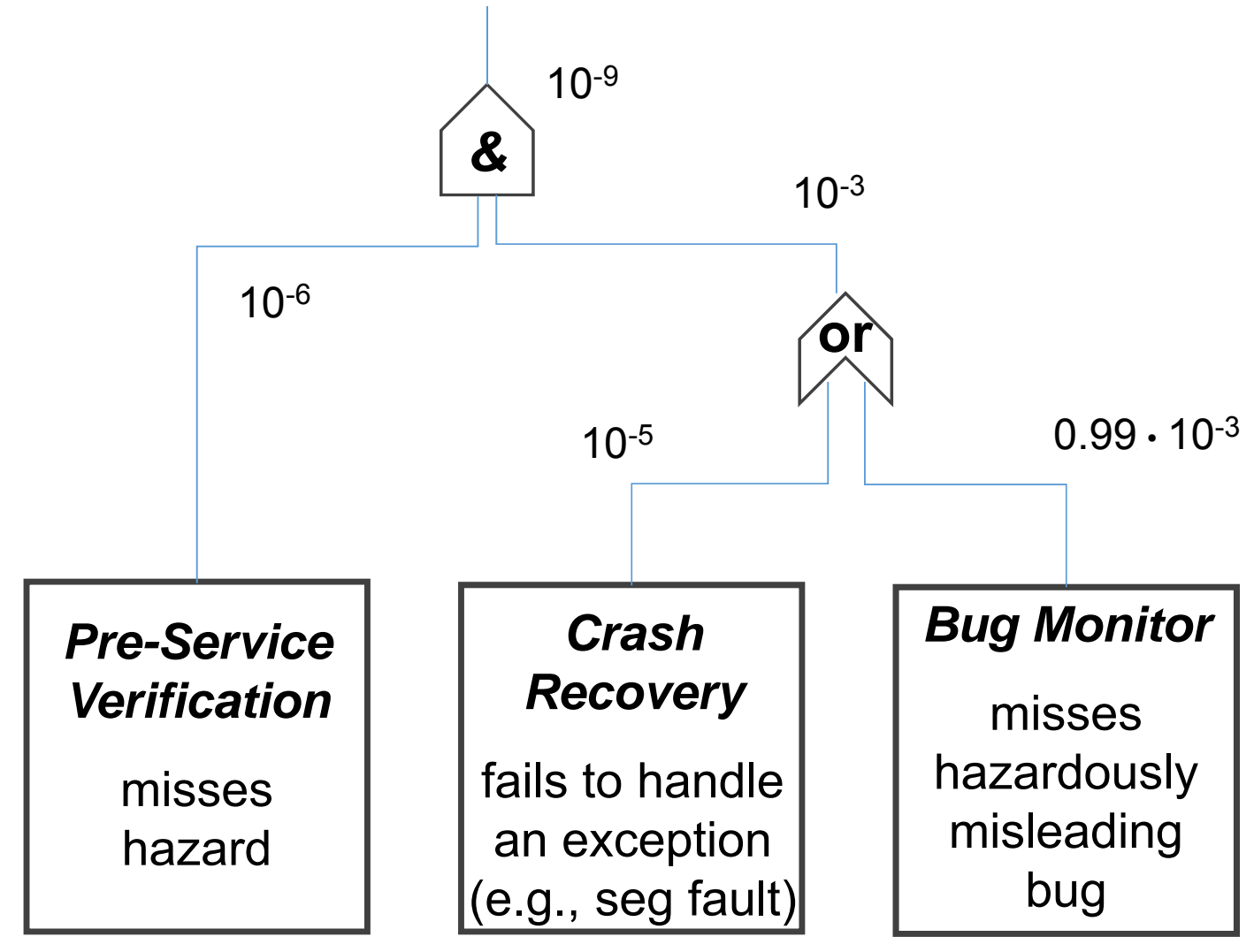


Context: Monitor Function

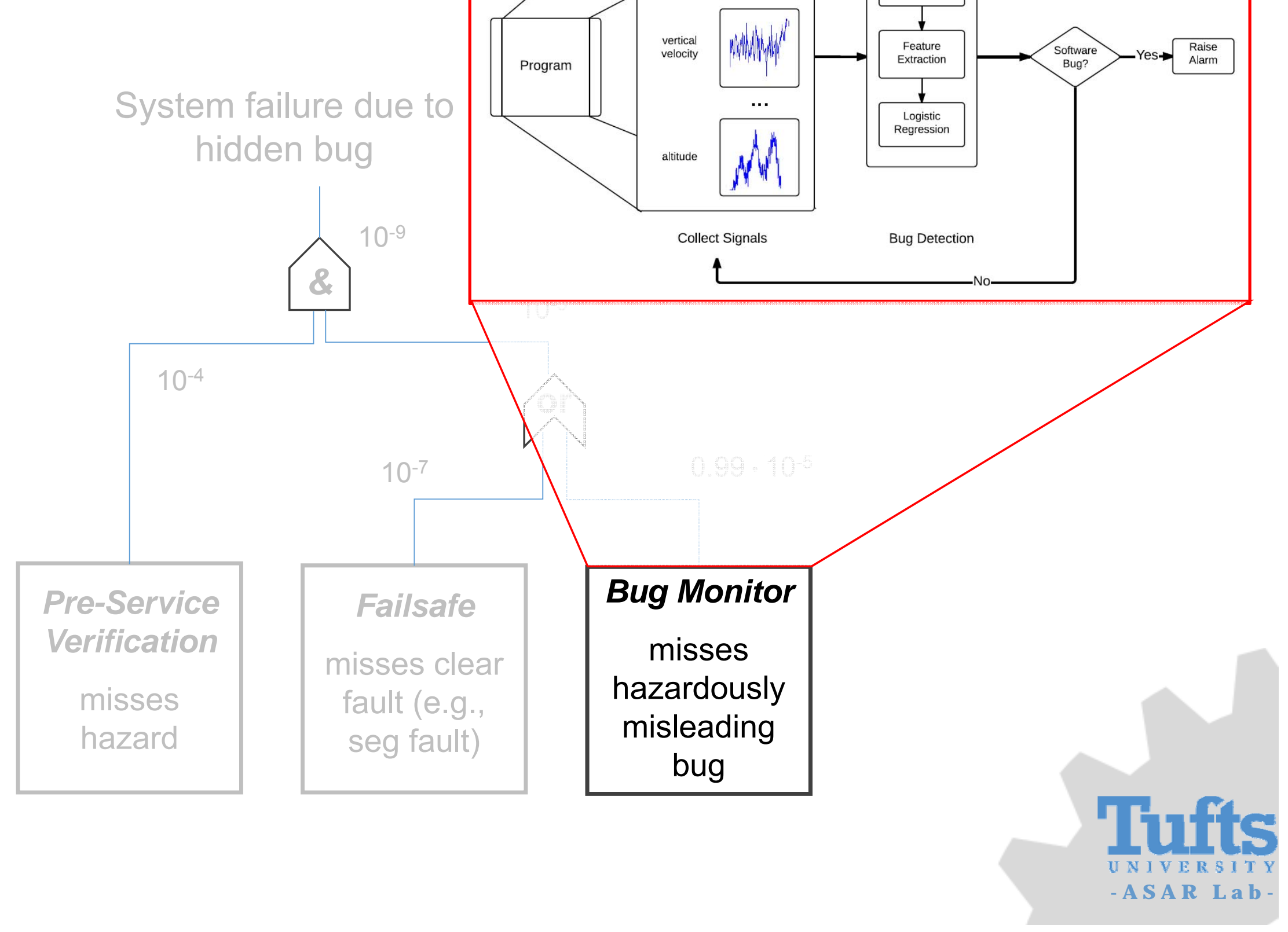




\section{Monitor Performance}

- Alarm rate: Rate of both false and true alarms

-Bug-detection envelope: Minimum "magnitude" bug that can reliably be detected

- Loss-of-integrity risk: Rate of undetected, severe bugs given pre-service verification and monitoring 


\section{Modeling Monitor to Quantify Performance}

- Monitor looks for

- Bugs that appear infrequently and are difficult to find ("Heisenbugs")

- Monitor warns of anomalies in real time

- Test statistic compares machine-learned model to code outputs

- Alarm occurs if statistic exceeds threshold

- As a starting point, simplify model of test-statistic noise

- Assume distribution unchanged when bias active, except for mean

- For approximate quantification only, assume a 1D Gaussian distribution
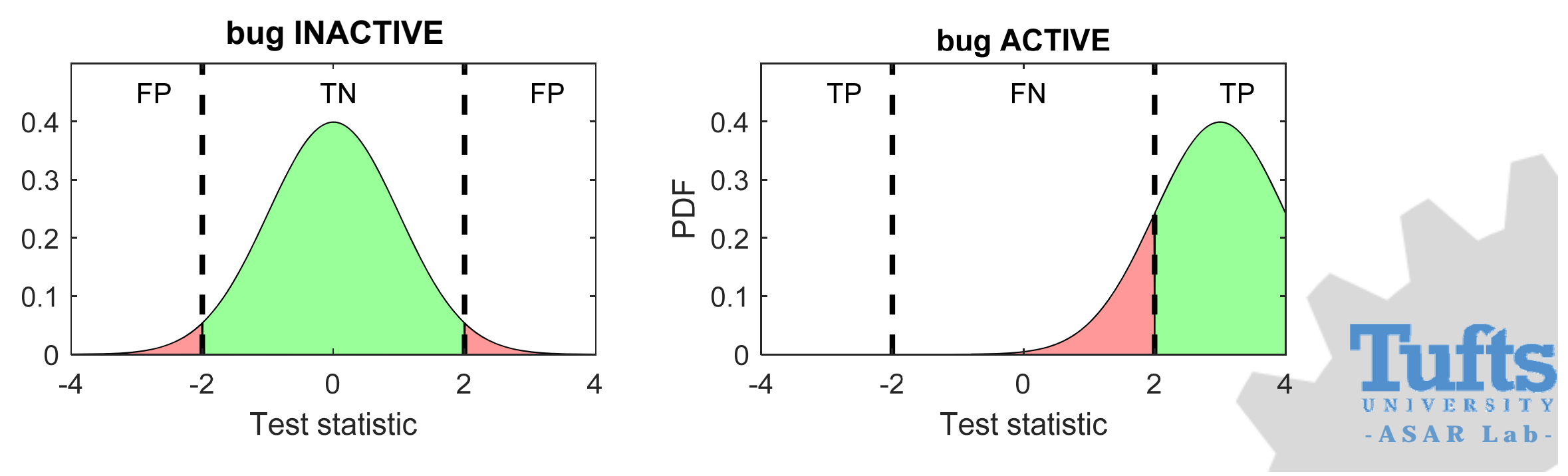


\section{Performance Characterization}

- Alarm rate:

$$
P_{a}=P_{v f}\left(1-P_{F N}\right)+\left(1-P_{v f}\right) P_{F P}
$$

- Detection envelope:

$$
\mu=\operatorname{solve}\left[P_{F N}=\int_{-T}^{T} p(x-\mu) d x\right]
$$

- Loss-of-integrity risk: $\quad P_{L O I}=P_{v f} P_{F N}$

Threshold: $T=$ solve $\left[P_{F P}=1-\int_{-T}^{T} p(x) d x\right]$ 


\section{Problem parameterized by}

$$
\begin{array}{cl}
P_{a}=\underline{P_{v f}}\left(1-\underline{P_{F N}}\right)+\left(1-\underline{P_{v f}}\right) \underline{P_{F P}} & P_{L O I}=\underline{P_{v f}} \underline{P_{F N}} \\
\mu=\operatorname{solve}\left[\underline{\left.P_{F N}=\int_{-T}^{T} p(x-\mu) d x\right]}\right. & T=\operatorname{solve}\left[\underline{P_{F P}}=1-\int_{-T}^{T} p(x) d x\right]
\end{array}
$$

- $P_{\mathrm{FP}}$ : False-positive rate

- $P_{\mathrm{FN}}$ : False-negative rate

- $P_{\text {vf }}$ : Verification fault probability 


\section{Design Problem}

1

- Find an acceptable set of output parameters $\left(P_{a}, \mu, P_{L O I}\right)$

- Selecting from space of input parameters $\left(P_{F P}, P_{F N}\right)$.

- Note: Low sensitivity to $P_{v f}$ if $P_{v f}<0.8 P_{a}$

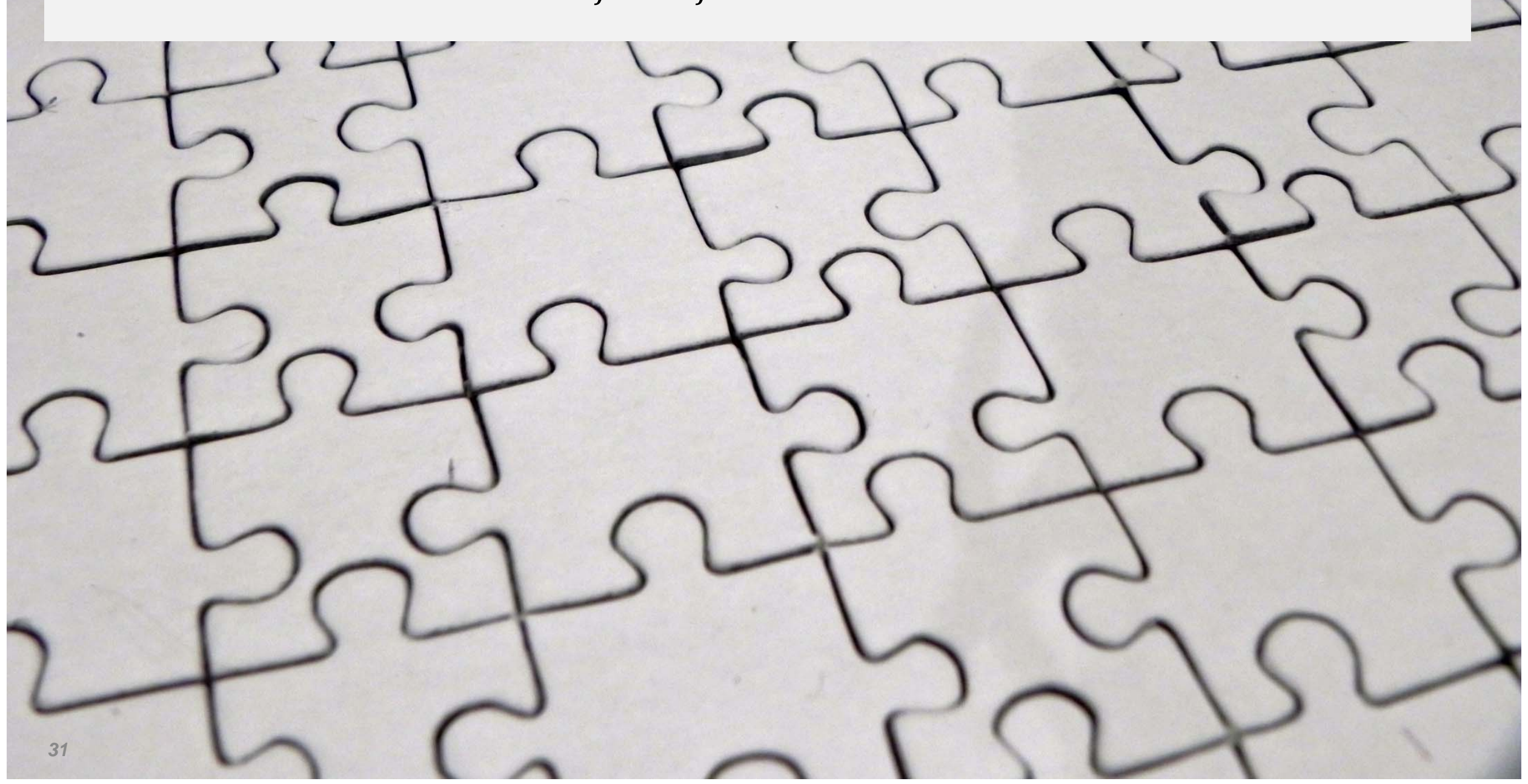




\section{Achievable Designs}

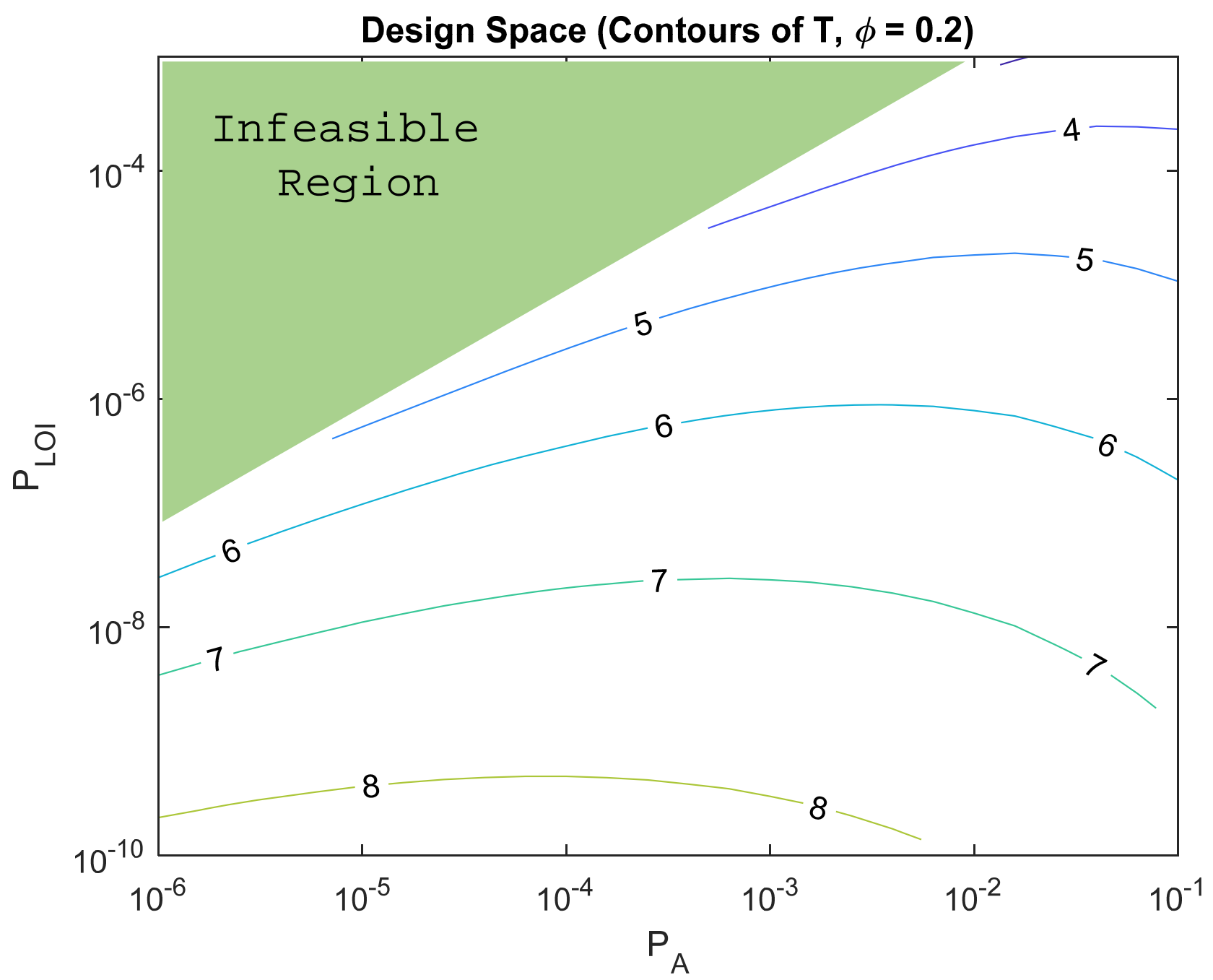

Contours relate three performance criteria:

$$
\mu=f\left(P_{a}, P_{L O I}\right)
$$

For details see: Rife, Hu, and Guyer. ION GNSS+ 2018. 


\section{Summary}

- Bug monitoring demonstrated for real bugs in flight software

- 70\% sensitivity demonstrated using snapshot monitor and (!) random probes

- Reasonable to infer higher sensitivity possible with guided probe selection and/or sequential monitoring

- Relaxing verification seems possible, even with low-sensitivity monitors

- The Gaussian probability model is clearly very approximate

- New challenge: Showing software robust to "small bugs"

- Bottom line: For a given level of safety (e.g. loss-of-integrity probability), preservice verification can be relaxed by carefully introducing bug monitoring 


\section{Acknowledgements}

\section{Tufts T) RedLine}

Support for research provided by NSF (NSF-1329341 and NSF-1836942) 